\title{
The Best Guess Approach to Phase I Trial Design
}

Daniela D. Rosa, John Harris, and Gordon C. Jayson

\author{
From the Cancer Research United King- \\ dom, Department of Medical Oncology, \\ Christie Hospital NHS Trust; School of \\ Law, University of Manchester, \\ Manchester, United Kingdom. \\ Submitted April 18, 2005; accepted \\ July 15, 2005 \\ Authors' disclosures of potential con- \\ flicts of interest and author contribu- \\ tions are found at the end of this \\ article. \\ Address reprint requests to Daniela D. \\ Rosa, Cancer Research United King- \\ dom, Department of Medical Oncology, \\ Christie Hospital, Wilmslow Rd, \\ Manchester M20 4BX, United Kingdom; \\ e-mail: dornellesrosa@hotmail.com. \\ C 2006 by American Society of Clinical \\ Oncology \\ 0732-183X/06/2401-206/\$20.00 \\ DOI: $10.1200 / J C O .2006 .02 .4299$
}

\section{HERE'S THE CASE}

A 56-year-old woman with advanced, incurable ovarian cancer was invited to be the first patient, in the first dose level, of a phase I trial of a new drug that was being given to patients for the first time. The patient information sheet stated that she was unlikely to benefit from her participation in the trial, and she was told that when phase I trials are initiated, the drug concentration is usually significantly less than the final recommended dose or maximumtolerated dose of the compound. The patient was concerned about not obtaining a tumor response with the lower dose level, and asked if she could delay participation until higher dose cohorts had opened for accrual.

At some point during the development of a new anticancer agent, the drug shifts from animals into humans. The first phase I trial conducted in humans aims to establish the toxicities associated with the drug, the maximum-tolerated dose, doselimiting toxicity, and pharmacokinetics. The need to establish a dose that is suitable for further evaluation in phase II clinical trials is of critical importance. ${ }^{1-3}$ Obtaining preliminary evidence of anti-tumor effect is a secondary goal in phase I studies. ${ }^{4,5}$

Patients who enroll onto phase I clinical trials of anticancer drugs have often exhausted all standard therapeutic options, and therefore, there is usually no alternative treatment for them. The conventional design of phase I trials in the cancer setting is to enroll a cohort, usually of three patients, who will be given the drug at a particular dose. If the cohort tolerates the drug without the development of significant toxicity then an additional cohort of patients is recruited. These patients are given a higher dose of the drug until a dose is reached in which a significant percentage of patients experience a dose-limiting toxicity (the point at which serious adverse effects appear so frequently as to render further dose escalation dangerous and burdensome). ${ }^{6}$ This allows a dose to be defined for phase II evaluation. ${ }^{7}$

One of the problems with patients taking part in first into human studies is that at the start of the trial the first dose is usually planned at a low level, often defined as that which is equivalent to one tenth of the dose which was lethal in $10 \%$ of rodents ${ }^{8,9}$ or one third of the minimum dose that causes any toxicity in dogs. The dose is taken from whichever is the more sensitive species. Further planned cohort doses are then increased, usually in a semi-logarithmic fashion, according to a modified Fibonacci series, ${ }^{10}$ which was designed for DNAdamaging agents, not for molecularly targeted drugs. A crucial ethical question is whether this trial serves the interests of patients who enter the first dose level, as they will usually be exposed to a low level of drug, which from previous experience, is unlikely to be the maximum-tolerated dose of the compound. ${ }^{9}$ Indeed, some patients ask whether they should enter the trial at the lowest dose level or whether they would be better off deferring entry until more active doses are available. ${ }^{11}$ The information given to patients for these trials states that they probably will not benefit from taking part in the trial, this may particularly be the case for patients who are recruited to the first dose level. ${ }^{1,2}$ The question then arises as to whether phase I drug trial design should be altered to maximize the chances of benefit for individual trial subjects?

One approach that could be taken is to estimate the likely effective dose from similar drugs within the same class. For instance, in the case of monoclonal antibodies, it is known that most evaluated products are eventually administered at doses between 1 and $10 \mathrm{mg} / \mathrm{kg}$ (or equivalent) every 1 to 4 weeks. ${ }^{13-16}$ This best guess approach to choosing initial doses might be associated with increased chances of toxicity, but some patients with cancer would accept this, probably because of the risks of disease progression and disease-related death. After all, at the stage of entering a phase I trial many patients will have received at least two chemotherapy regimens and will have exhausted standard care options. Given the choice between an almost certainly subtherapeutic dose versus a dose that could be more likely to be therapeutic, but also more likely to be toxic, it is possible that the majority of patients would prefer the dose more likely to be therapeutic. If they do have this preference and are fully informed, it is difficult to see why they should not be offered the dose more likely to benefit them. 
This view that patients should be offered a choice between dose levels may serve more interests than that of the individual patient. It may benefit the study by removing unnecessary cohorts of patients treated at low doses, thereby shortening the time it takes to define the dose needed for phase II evaluation. It may save money for the drug company, reduce drug development time benefiting future patients treated with the trial drug, and potentially help additional patients by accelerating the development of other members of that family of drugs. It may also give the trial subjects a better chance of benefiting from trial participation, remembering that these are patients with little to lose and for whom the risk benefit ratio is both rational and prudent. At worst, they will have toxic adverse effects that might hasten death, although it was recently found that the overall rate of death due to toxic events in phase I trials was quite low, being around $0.5 \% .^{12,17}$ But, providing fully informed consent may be deemed a reasonable trade-off for people likely to be facing death in a matter of months unless a therapeutic agent can be found for them. So, far from being unethical to allow patients to run extra risk of death in the hope of extended life or palliation of symptoms, it might be less ethical to expect the sacrifices entailed in trial participation while offering less chance of benefit than is possible. It would equally be less ethical not to hasten development of beneficial drugs if we can do so while preserving the ethical and scientific integrity of the trial.

Much of the drug development work around the world involves pharmaceutical companies in one way or another. Naturally these companies are both motivated to produce drugs that help the general population, and which will increase the profit for their company. If a new drug enters early clinical trial evaluation and is associated with a significant toxicity in a first into human study, then it is possible that the development of the drug could be impeded. If a best guess approach was taken with the patient's consent, there might be an increased risk of toxicity early in the product's development, which could lead to litigation and compromise both the profits of the company and the timely development of a useful product. However, neither of these outcomes is a necessary consequence of the current proposal. On the contrary, if all compounds were developed in this way, and the best guess approach was accepted as both ethical and good practice, risks of litigation and bad publicity would be minimized. Perhaps one distinction that should be made is that between first class drugs in which there is no precedent to guide dosing versus new drugs in which related compounds have already entered the clinical practice. ${ }^{1}$ These factors would influence what constitutes the best guess, but not the ethical arguments for adopting the best guess approach.

Predicting an active starting dose may not be appropriate when there are no analogs or members of the same class (eg, antibodies). ${ }^{18}$ In this situation, one alternative approach for patients who enter a classically designed phase I study of anticancer drugs at the lowest dose level is that either the dose is escalated for that patient (intrapatient dose escalation) to maximize the chances of benefit or the patient is allowed to re-enter the study at a higher dose level, depending on issues such as the continued fitness of the patient to re-enter the study or the immune response to the compound. ${ }^{19,20}$ Indeed, recent studies using an accelerated titration design (ie, rapid intrapatient drug dose escalation) have demonstrated acceptable toxicity profiles. ${ }^{21-30}$

Previously proposed methods to address subtherapeutic starting doses were based on in vitro and in vivo preclinical pharmacokinetic data and allometric scaling to predict in vivo drug clearance in hu- mans. ${ }^{31,32}$ However, this dose escalation process has little scientific rationale and requires too many steps that expose a fair number of patients to low and presumably ineffective therapy. ${ }^{33}$ While interpatient variability remains a problem that might be compounded using the best guess approach, this could be reduced by Bayesian methods. ${ }^{10,17,34,35}$ However, the benefit of this proposal would be an accelerated progression towards phase II trials, the design of which has been modified for the modern targeted therapies where larger randomized phase II trials have been used to highlight the best dose. Furthermore, the power of pharmacodynamic evaluation might be better focused in the randomized phase II setting where interpatient variation is reduced by recruiting large cohorts of patients to particular dose levels. If this approach also hastens the general availability of beneficial new therapies, then not only future trial subjects but also large numbers of future patients may benefit from an educated guess.

\section{REFERENCES}

1. Kurzrock R, Benjamin RS: Risks and benefits of phase 1 oncology trials, revisited. N Engl J Med 352:930-932, 2005

2. Horng $S$, Emanuel EJ, Wilfond $B$, et al: Descriptions of benefits and risks in consent forms for phase I oncology trials. N Engl J Med 347:2134-2140, 2002

3. Nottage M, Siu L: Principles of clinical trial design. J Clin Oncol 20:42s-46s, 2002

4. Miller M: Phase I cancer trials: A crucible of competing priorities. Int Anesthesiol Clin 39:13-33, 2001

5. Meropol NJ, Weinfurt KP, Burnett CB, et al: Perceptions of patients and physicians regarding phase I cancer clinical trials: Implications for physicianpatient communication. J Clin Oncol 21:2589-2596, 2003

6. Conaway MR, Dunbar S, Peddada SD: Designs for single- or multiple-agent phase I trials. Biometrics 60:661-669, 2004

7. Orlowski RZ, Stinchcombe TE, Mitchell BS, et al: Phase I trial of the proteasome inhibitor PS-341 in patients with refractory hematologic malignancies. J Clin Oncol 20:4420-4427, 2002

8. Eisenhauer EA, O'Dwyer PJ, Christian M, et al: Phase I clinical trial design in cancer drug development. J Clin Oncol 18:684-692, 2000

9. Newell DR, Silvester J, McDowell C, et al: The Cancer Res UK experience of pre-clinical toxicology studies to support early clinical trials with novel cancer therapies. Eur J Cancer 40:899-906, 2004

10. Bolognese JAA: Monte Carlo comparison of three up-and down designs for dose ranging. Control Clin Trials 4:187-196, 1983

11. Agrawal M, Emanuel EJ: Ethics of phase I oncology studies. JAMA 290:1075-1082, 2003

12. Horstmann $E, M c C a b e$ MS, Grochow $L$, et al: Risks and benefits of phase 1 oncology trials, 1991 through 2002. N Engl J Med 352:895-904, 2005

13. Saltz LB, Meropol NJ, Loehrer PJ, et al: Phase II trial of cetuximab in patients with refractory colorectal cancer that expresses the epidermal growth factor receptor. J Clin Oncol 22:1201-1208, 2004

14. Slamon DJ, Leyland-Jones $B$, Shak $S$, et al: Use of chemotherapy plus a monoclonal antibody against HER2 for metastatic breast cancer that overexpresses HER2. N Engl J Med 344:783-792, 2001

15. Willett CG, Boucher Y, di Tomaso E, et al: Direct evidence that the VEGF specific antibody bevacizumab has antivascular effects in human rectal carcinoma. Nat Med 10:145-147, 2004

16. Coiffier B, Lepage E, Briere J, et al: CHOP chemotherapy plus rituximab with $\mathrm{CHOP}$ chemotherapy alone in elderly patients with diffuse large-B-cell lymphoma. N Engl J Med 346:235-242, 2002

17. Quigley J, Chevret S: Methods for dose-finding studies in cancer clinical trials: A review and results of a Monte Carlo study. Stat Med 10:1647-1664, 1991

18. Korn EL, Arbuck SG, Pluda JM, et al: Clinical trial designs for cytostatic agents: Are new approaches needed? J Clin Oncol 19:265-272, 2001

19. Storer BE: Design and analysis of phase I clinical trials. Biometrics 45:925-937, 1989

20. Simon $R$, Freidlin $B$, Rubinstein $L$, et al: Accelerated titration designs for phase I clinical trials in oncology. J Natl Cancer Inst 89:1138-1147, 1997

21. Grem JL, Morrison G, Guo XD, et al: Phase I and pharmacologic study of 17-(allylamino)-17-demethoxygeldanamycin in adult patients with solid tumors. J Clin Oncol 23:1885-1893, 2005

22. Goetz MP, Toft D, Reid J, et al: Phase I trial of 17-allylamino-17demethoxygeldanamycin in patients with advanced cancer. J Clin Oncol 23:10781087,2005 
23. Wadler S, Makower D, Clairmont $C$, et al: Phase I and pharmacokinetic study of the ribonucleotide reductase inhibitor, 3-aminopyridine-2-carboxaldehyde thiosemicarbazone, administered by 96 -hour intravenous continuous infusion. J Clin Oncol 22:1553-1563, 2004

24. Matsumura $Y$, Hamaguchi $T$, Ura $T$, et al: Phase I clinical trial and pharmacokinetic evaluation of NK911, a micelle-encapsulated doxorubicin. $\mathrm{Br} \mathrm{J}$ Cancer 91:1775-1781, 2004

25. Syed $S$, Takimoto $C$, Hidalgo $M$, et al: A phase I and pharmacokinetic study of col-3 (metastat), an oral tetracycline derivative with potent matrix metalloproteinase and antitumor properties. Clin Cancer Res 10:6512-6521, 2004

26. Anderson HL, Yap JT, Miller MP, et al: Phase I and pharmacokinetic study of Bibx 1382 Bs: An epidermal growth factor receptor (EGFR) inhibitor, given in a continuous daily oral administration. J Clin Oncol 21:2823-2830, 2003

27. Gadgeel SM, Boinpally RR, Heilbrun LK, et al: A phase I clinical trial of spicamycin derivative KRN5500 (NSC 650426) using a phase I accelerated titration "2B" design. Invest New Drugs 21:63-74, 2003

28. Plummer R, Ghielmini $M$, Calvert $P$, et al: Phase I and pharmacokinetic study of the new taxane analog BMS-184476 given weekly in patients with advanced malignancies. Clin Cancer Res 8:2788-2797, 2002
29. Dittrich C, Greim G, Borner M, et al: Phase I and pharmacokinetic study of BIBX 1382 BS, an epidermal growth factor receptor (EGFR) inhibitor, given in a continuous daily oral administration. Eur J Cancer 38:1072-1080, 2002

30. Goh BC, Ratain MJ, Bertucci D, et al: Phase I study of ZD9331 on short daily intravenous bolus infusion for 5 days every 3 weeks with fixed dosing recommendations. J Clin Oncol 19:1476-1484, 2001

31. Houston JB: Utility of in vitro drug metabolism data in predicting in vivo metabolic clearance. Biochem Pharmacol 47:1469-1479, 1994

32. Lave $T$, Coassolo $P$, Reigner $B$ : Prediction of hepatic metabolic clearance based on interspecies allometric techniques and in vitro-in vivo correlations. Clin Pharmacokinet 36:211-231, 1999

33. Christian MC, Korn EL: The limited precision of phase I trials. J Natl Cancer Inst 86:1662-1663, 1994

34. Quigley J, Pepe M, Fisher L: Continual reassessment method: A practical design for phase I clinical trials in cancer. Biometrics 46:33-48, 1990

35. Quigley J: Estimating the probability of toxicity at the recommended doses following a phase I clinical trial in cancer. Biometrics 48:853-862, 1992

\section{Authors' Disclosures of Potential Conflicts of Interest}

The authors indicated no potential conflicts of interest.

\section{Author Contributions}

Conception and design: Daniela D. Rosa, John Harris, Gordon C. Jayson

Manuscript writing: Daniela D. Rosa, John Harris, Gordon C. Jayson 